\title{
Casimir Force in Confined Polymer Blends or Ternary Polymer Solutions
}

\author{
M. Benhamou, M. El Yaznasni, H. Ridouane, and E.-K. Hachem \\ Laboratoire de Physique des Polymères et Phénomènes Critiques \\ Faculté des Sciences Ben M'sik, B.P. 7955, Casablanca, Morocco
}

Received on 4 May, 2006

\begin{abstract}
This paper is devoted to a review of recent progresses concerning the computation of the Casimir force between two parallel plates delimitating a polymer blend or a ternary polymer solution (with a good solvent). We assume that, close to the consolute point, one or the two polymers of the mixture are strongly attracted by the plates (critical adsorption). For both systems, the induced force originates from the fluctuations of composition near the consolute point. In polymer blends case, it was found that the force decreases with separation $L$ between the two plates as $L^{-4}$, with a known universal amplitude. For ternary polymer solutions, however, it has been shown that the interaction force decays rather as $L^{-3}$. This drastic change of the force expression is due to the presence of the good solvent, which gives rise to additional fluctuations of polymer concentration. To do calculations, for the two systems, use is made of the standard $\varphi^{4}$-theory, where the field $\varphi$ is the order parameter or composition fluctuation.
\end{abstract}

Keywords: Polymer mixtures; Confinement; Casimir Forces

\section{INTRODUCTION}

The computation of the interaction forces induced by the fluctuations of some physical entity in confining geometries is an old problem. The first investigation was due to Casimir [1], who discovered that the vacuum fluctuations of a confined electromagnetic field generate an attractive force between two parallel uncharged conducting plates, which are a finite distance $L$ apart. This is the Casimir effect [2], which has received its final confirmation in recent experiments $[3,4]$.

Afterwards, Fisher and de Gennes [5] remarked that an analogous effect also appears in the context of Statistical and Condensed Matter Physics, for those critical systems restricted by boundaries. In this case, the critical fluctuations of the order parameter play the role of the vacuum quantum fluctuations.

Confined critical fluids, such as a fluid near the liquid-gas critical point, a binary liquid near the consolute point, ${ }^{4} \mathrm{He}$ liquid near the $\lambda$-point, generate long-range forces between the confining walls. These forces, termed critical Casimir forces in literature [6], obey universal scaling functions [7-10]. At criticality, these scaling functions reduce to universal amplitudes multiplying a negative power law in separation $L$ between the confining walls $[7-10]$. From a theoretical point of view, the critical Casimir forces have been extensively investigated, using conformal invariance in $d=2[11-15]$, Renormalization-Group (RG) in $d=4-\varepsilon[6,9,10,16-21]$, and Monte Carlo (MC) simulations [22]. Experimentally, confined critical fluids or colloids immersed in binary liquid mixtures have been the subject of numerous investigations [23].

Very recently [24], one has shown the existence of some universal force within critical polymer blends confined to two parallel plates, which strongly adsorb polymers near the critical point $T_{c}$. This is the so-called critical adsorption $[5,25-35]$. It was found [24] that this force, $\Pi$, decays with thickness $L$ according to a negative power law, that is $\Pi \sim L^{-4}$, with a known universal amplitude. These results were extended to ternary solutions made of two chemically incompatible polymers immersed in a good solvent, and restricted by the same boundaries [36]. Of course, the presence of the good solvent affects the expression of the induced force. It has been shown [36] that the latter decreases rather as $\Pi \sim L^{-3}$.

In this paper, we review recent trends concerning the computation of the Casimir force between two parallel plates delimitating a polymer blend or a ternary polymer solution. To this end, use will be made of the $\varphi^{4}$-field theory, where the field $\varphi$ is nothing else but the composition fluctuation.

The remainder of the presentation proceeds as follows. In Sec. II, we present the computation of the induced force of a confined binary polymer mixture. Extension to ternary polymer solutions is the aim of Sec. III. We draw some concluding remarks in the last section.

\section{CASIMIR FORCE IN CONFINED POLYMER BLENDS}

Consider a critical mixture made of two incompatible polymers $A$ and $B$ confined between two parallel homogeneous plates 1 and 2, which are separated by a finite distance $L$. We assume that the thickness $L$ is much smaller than the bulk thermal correlation length of the mixture $\xi_{t}^{0} \sim$ $a \sqrt{N}\left|1-T_{c} / T\right|^{-1 / 2}$, that is $L<<\xi_{t}^{0}$. Thus, we are concerned with a film geometry. There, $N$ is the common polymerization degree of polymer chains and $a$ the monomer size. We suppose that, close to the consolute point, one or the two polymers prefer to be attracted by the plates (critical adsorption). A point of the medium can be described by a three-dimensional vector $\mathbf{r}=(\rho, z)$, where $z \in[0, L]$ is the distance from the plate 1 taken as origin and $\rho=(x, y)$ the two-dimensional parallel vector. To describe the physics of the system, we introduce an order parameter (or composition fluctuation) $\varphi=\Phi_{A}-\Phi_{B}$, which is a scalar field depending on the considered point $\mathbf{r}$. Here, $\Phi_{A}$ is the monomer fraction of polymer $A$, and $\Phi_{B}=1-\Phi_{A}$ that of polymer $B$. We denote by $\varphi_{1}(\rho)$ and $\varphi_{2}(\rho)$ the values of $\varphi$-field on plates 1 and 
2 , respectively. Because of the homogeneity property of the two plates, the order parameter $\varphi$ depends only on the perpendicular distance $z$. The translational symmetry property along the parallel directions implies that the surface composition fluctuations are independent on the considered point, and they will be simply denoted by $\varphi_{1}$ and $\varphi_{2}$. We shall be concerned only with two special cases : symmetric or asymmetric plates. For symmetric plates, we have the same composition on both sides, and we will set $\varphi_{1}=\varphi_{2} \equiv \varphi_{0}$. While for asymmetric plates, we have opposite surface compositions, and we will set $\varphi_{1}=-\varphi_{2} \equiv-\varphi_{0}$.

The confined polymer mixture can be described by the following free energy functional (per unit area) [24]

$$
\frac{F[\varphi]}{\mathcal{A} k_{B} T}=f_{1}\left(\varphi_{1}\right)+f_{2}\left(\varphi_{2}\right)+a^{-3} \int_{0}^{L} d z\left[G(\varphi)+\kappa\left(\frac{d \varphi}{d z}\right)^{2}\right],
$$

where $\mathcal{A}$ is the plates area, $T$ the absolute temperature and $k_{B}$ the Boltzmann constant. The first two terms $f_{1}\left(\varphi_{1}\right)$ and $f_{2}\left(\varphi_{2}\right)$ account for the contributions of the surfaces to the free energy. Their forms will be given below. The last one accounts for the bulk contribution, with $G(\varphi)$ the bulk free energy density [37],

$$
G(\varphi)=\frac{\chi_{c}-\chi}{4} \varphi^{2}+\frac{1}{12 N} \varphi^{4},
$$

which is the expansion to fourth order of the standard FloryHuggins (FH) free energy [37,38], in the vicinity of the critical monomer fraction $\Phi_{c}=1 / 2$, or equivalently around $\varphi=0$. In the above expression, $\chi$ is the standard Flory interaction parameter, which is inversely proportional to the absolute temperature $T$, and $\chi_{c}=2 / N$ its critical value. In relation (1), $\kappa=a^{2} / 9$ is a constant. The surface contribution is that usually encountered in surface critical phenomena $[39,40]$, that is

$$
f_{1}\left(\varphi_{1}\right)+f_{2}\left(\varphi_{2}\right)=\sum_{i=1,2}\left(-h_{i} \varphi_{i}+\frac{c_{i}}{2} \varphi_{i}^{2}\right) .
$$

In this equality, $h_{i}$ 's are the surface chemical potentials differences of the two polymers, and $c_{i}$ 's the surface coupling constants measuring the interaction strength of $A$ and $B$ monomers with plates. Let us discuss these surface parameters. First, the surface coupling constant is the same for symmetric and asymmetric plates, and will be simply denoted by $c \equiv c_{1}=c_{2}$. For the symmetric case, the surface field has the same values on both surfaces, and will be denoted by $h \equiv h_{1}=h_{2}$. While for the asymmetric one, the fields on the two sides are opposite, that is $h \equiv-h_{1}=h_{2}$. The critical adsorption emerges in the limit $h \rightarrow \infty$, at fixed surface coupling constant $c>0$. This is equivalent to impose the condition : $\varphi_{0} \rightarrow \infty$, which is the fixed point of the so-called normal transition [40].

The equilibrium profile, $\varphi(z)$, can be obtained through a standard minimization of the above total free energy, that is $\delta F / \delta \varphi=0$. Then, the profile is solution to the following nonlinear differential equation

$$
\frac{\chi_{c}-\chi}{2} \varphi^{2}+\frac{1}{3 N} \varphi^{3}-2 \kappa \frac{d^{2} \varphi}{d z^{2}}=0,
$$

together with the following boundary conditions

$$
2 a^{-3} \kappa\left[\frac{d \varphi}{d z}\right]_{z=0}=c \varphi_{0}-h, \quad 2 a^{-3} \kappa\left[\frac{d \varphi}{d z}\right]_{z=L}=-c \varphi_{0}+h,
$$

for symmetric plates, or

$$
2 a^{-3} \kappa\left[\frac{d \varphi}{d z}\right]_{z=0}=c \varphi_{0}-h, \quad 2 a^{-3} \kappa\left[\frac{d \varphi}{d z}\right]_{z=L}=c \varphi_{0}-h,
$$

for asymmetric ones.

The first integral of the above differential equation is

$$
\kappa\left(\frac{d \varphi}{d z}\right)^{2}=G(\varphi)+C,
$$

where $C$ is an integration constant that depends on the bulk parameters, the surface ones $c$ and $h$ and the separation $L$. For symmetric plates where $\varphi(0)=\varphi(L)=\varphi_{0}$, the expected profile exhibits a minimum point at the middle of the film [41], that is at $z=L / 2$. In this case, the integration constant $C$ is directly related to the value of the FH free energy density $G\left(\varphi_{m}\right)$, that is $C=-G\left(\varphi_{m}\right)$. Here, $\varphi_{m}=\varphi(L / 2)$ is the minimal value of the equilibrium profile. For asymmetric plates where $\varphi(0)=-\varphi(L)=\varphi_{0}$, it becomes rather a monotonously decreasing function from $z=0$ to $z=L$.

We have now all necessary ingredients for the determination of the induced force, which results from the fluctuations of composition, that are strong near the critical point. To this end, we first consider the symmetric case, for which the equilibrium profile is solution to the differential equation (4) with boundary conditions (5). The starting point is the first integral defined by relation (7), with $C=-G\left(\varphi_{m}\right)$. It is easy to show, from this relation, that the minimal value $\varphi_{m}$ of the equilibrium profile is given by the quadrature formula

$$
L=2 \int_{\varphi_{m}}^{\varphi_{0}} \sqrt{\frac{\kappa}{G(\varphi)-G\left(\varphi_{m}\right)}} d \varphi,
$$

where the function $G(\varphi)$ is taken at the critical point, that is $G(\varphi)=\varphi^{4} / 12 N$. The above relation expresses the dependence of the minimal value $\varphi_{m}$ on the film thickness $L$ and surface composition $\varphi_{0}$. On the other hand, the attractive force (per unit area), $\Pi_{a}$, is given by the first derivative of the total free energy, with respect to separation $L$, that is $\Pi_{a}=-\left(1 / \mathcal{A} k_{B} T\right) \partial F / \partial L$. It has been shown [41] that this force is simply given by

$$
\Pi_{a}=-\frac{k_{B} T_{c}}{a^{3}} G\left(\varphi_{m}\right) .
$$

Then, the induced force is directly proportional to the integration constant $C$, that is $\Pi_{a}=k_{B} T_{c} a^{-3} C$. Since $G\left(\varphi_{m}\right)$ is positive definite, the force is attractive. In addition, relation (9) indicates that the induced force is simply given by the knowledge of the minimal value $\varphi_{m}$ of the profile.

Come back to quadrature formula (8) and make the variable change : $\varphi \rightarrow x=\varphi / \varphi_{m}$, to get

$$
\frac{L}{a \sqrt{N}}=\frac{4}{\sqrt{3}} \frac{1}{\varphi_{m}} \int_{1}^{\varphi_{0} / \varphi_{m}} \frac{d x}{\sqrt{x^{4}-1}} .
$$


In the critical adsorption limit, the upper bound of the above integral goes to infinity, that is $\varphi_{0} / \varphi_{m} \rightarrow \infty$, and one finds the following exact expression for the interaction force [24]

$$
\Pi_{a}=\frac{N k_{B} T_{c}}{a^{3}} \frac{\Delta_{\uparrow \uparrow}}{(L / a)^{4}},
$$

with the universal amplitude

$$
\Delta_{\uparrow \uparrow}=-\frac{[\Gamma(1 / 4)]^{8}}{432 \pi^{2}} \simeq-7.002693379 \text {, }
$$

where $\Gamma(z)$ is the gamma function [42].

For asymmetric plates, the profile is solution to the differential equation (4) with the boundary conditions (6). The associated force is directly proportional to the integration constant $C$ as in symmetric case, that is $\Pi_{r}=k_{B} T_{c} a^{-3} C[24,41]$. Since $C$ is positive $[24,41]$, this force is repulsive. To obtain its scaling behavior, one can follow the same techniques as above. We recall simply the result $[24]$

$$
\Pi_{r}=\frac{N k_{B} T_{c}}{a^{3}} \frac{\Delta_{\uparrow \downarrow}}{(L / a)^{4}},
$$

with the universal amplitude

$$
\Delta_{\uparrow \downarrow}=\frac{[\Gamma(1 / 4)]^{8}}{108 \pi^{2}} \simeq 28.01077353 .
$$

Now, let us comment the above results.

Firstly, we note that both attractive and repulsive forces decay with separation $L$ according to the same power law, that is $\Pi_{a, r} \sim \mp L^{-4}$.

Secondly, when these forces are reduced by the $N k_{B} T_{c} a^{-3}$. factor, they exhibit a universal behavior, and depend only on film thickness $L$ and not on surface interactions details (through $c$ and $h$ ). The surface parameters $c$ and $h$ contribute only to corrections to the leading critical behavior. In addition, the corresponding amplitudes $\Delta_{\uparrow \uparrow}$ and $\Delta_{\uparrow \downarrow}$ are pure numbers.

Thirdly, as it should be, these forces are proportional to the polymerization degree $N$ of chains.

Fourthly, remark that this power law in $L$ resembles that defining the electromagnetic Casimir force in the same geometry [1].

Sixthly, notice that, intuitively, the repulsive force must be stronger than the attractive one, for the same separation $L$. Indeed, this is due to the fact that, for opposite plates, the mixture may be regarded as two lamellar phases alternatively rich is $A$ and $B$-polymers. Quantitatively, one has the ratio

$$
\left|\frac{\Pi_{r}}{\Pi_{a}}\right|=4
$$

which is a pure integer number, independently on separation $L$. This same relation shows that the repulsive force is four times more intense than the attractive one.

Finally, attractive and repulsive forces versus distance $L$ are depicted in Figs. 1 and 2, respectively.

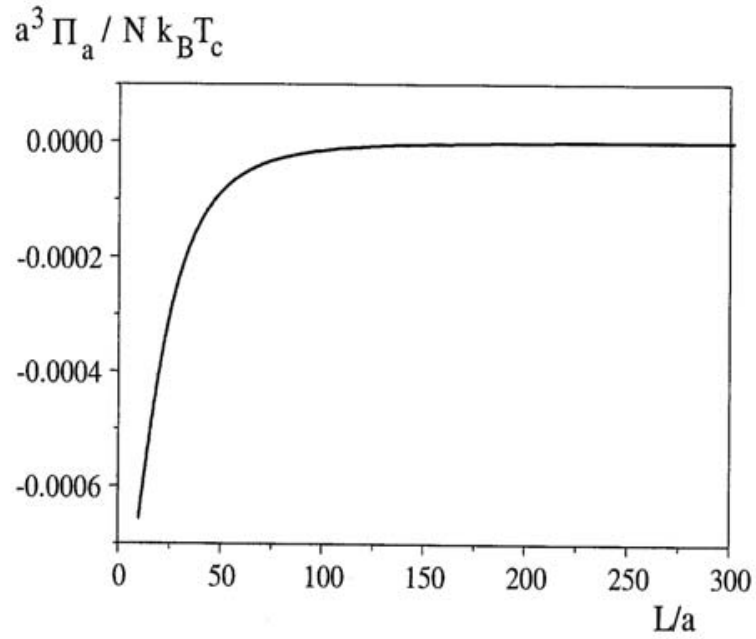

FIG. 1: The reduced attractive induced force $a^{3} \Pi_{a} / N k_{B} T_{c}$ versus the separation $L$ (expressed in monomer size $a$ units).

$$
\mathrm{a}^{3} \Pi_{\mathrm{r}} / \mathrm{Nk}_{\mathrm{B}} \mathrm{T}_{\mathrm{c}}
$$

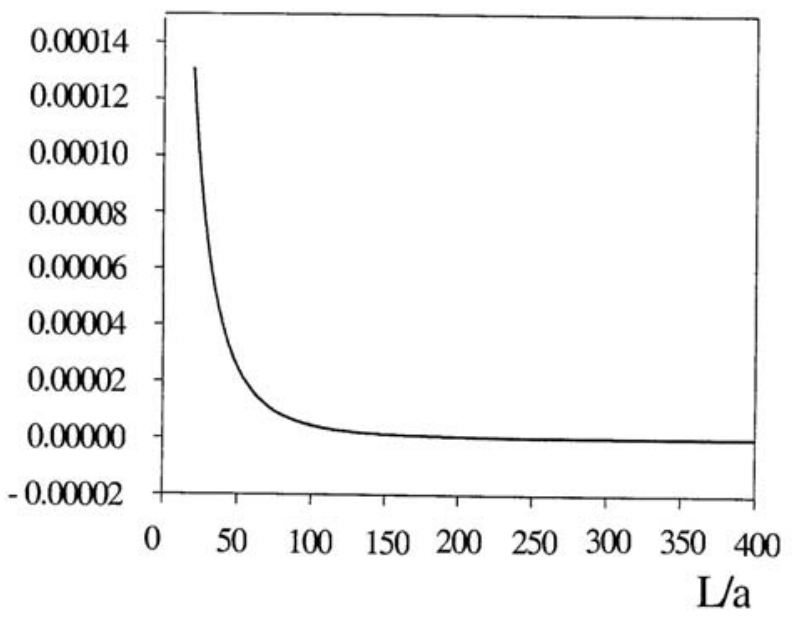

FIG. 2: The reduced repulsive induced force $a^{3} \Pi_{r} / N k_{B} T_{c}$ versus the separation $L$ (expressed in monomer size $a$ units).

\section{CASIMIR FORCE IN CONFINED TERNARY POLYMER SOLUTIONS}

Now, consider a mixture of two incompatible long polymers $A$ and $B$ dissolved in a common good solvent. We assume that the ternary polymer solution is confined between two adsorbing parallel plates 1 and 2, which are a finite distance $L$ apart. The latter is assumed to be smaller than the bulk thermal correlation length $\xi_{t}\left(L<<\xi_{t}\right)$. This characteristic length that will be defined below, measures the spatial extent of correlations. The opposite case where $L>>\xi_{t}$ contributes to the leading critical behavior only by exponentially decreasing small corrections $[7,43]$. We suppose that plates attract strongly one or the two polymers close to the consolute point (critical adsorption). As consequence, plates 
experience an effective force originating from the strong fluctuations of composition near the consolute point. Such a force depends on film thickness $L$ and the considered surface universality class. For the sake of simplicity, we will assume that either plates have the same preference to attract one component (symmetric plates) or they have an opposed preference (asymmetric plates). Besides the chemical segregation between unlike chains, one is in the presence of excluded volume interactions leading to swelling of polymer chains.

The fundamental problem to apprehend is a quantitative study of the influence of the good solvent on the force expression. Calculations will be done using, first, the blob model [44], and second the RG.

\section{A. Mean-field results}

To determine the induced force, we shall need the free energy. To get its expression, we start by recalling some useful background on the demixing transition in the presence of a good solvent of infinite extent.

Start with a mixture of two chemically incompatible polymers $A$ and $B$, dissolved in a common good solvent. This mixture may be polystyrene-poly(methylmethacrylate) in toluene or polystyrene-poly(dimethylsiloxane) in propylbenzene. For simplify, the two polymers $A$ and $B$ are assumed to have the same polymerization degree $N$ (monodisperse system). We denote by $\Phi=\Phi_{A}+\Phi_{B}$ the overall monomer fraction, where $\Phi_{A}$ and $\Phi_{B}$ are the respective monomer fractions of $A$ and $B$ polymers.

We recall that, in dilute solution, where the overall monomer fraction $\Phi$ is below the threshold $\Phi^{*} \sim N^{1-v d}(v \simeq$ 0.588 , in $d=3$ ) [37], $A$ and $B$-chains behave like separated swollen coils avoiding each other completely, and in principle no phase separation is expected. In semi-dilute solution $\left(\Phi^{*}<<\Phi<1\right)$, however, chains overlap and can be regarded as a sequence of uncorrelated subunits or blobs of types $A$ and $B$. Each chain contains $Z(\Phi) \sim N \Phi^{1 /(v d-1)}$ blobs. The blob size or screening length [37], $\xi(\Phi)$, depends only on the total monomer fraction $\Phi$, and scales as [37] : $\xi(\Phi) \sim \Phi^{v /(1-v d)}$, where $a$ is the monomer size.

Using field-theoretical RG, it has been shown [44] that, for a high-molecular-weight solution, a given chain cannot distinguish between an $A$ and $B$-chain. This means that the chemical difference is irrelevant, and manifests itself only in correction to the leading behavior of the osmotic pressure. In fact, these corrections are important and govern thermodynamics of the demixing transition. From the obtained expression of the osmotic pressure, the authors of Ref. [44] derived the following expression for the free energy

$$
\frac{F_{0}}{k_{B} T}=\frac{x}{Z(\Phi)} \ln x+\frac{1-x}{Z(\Phi)} \ln (1-x)+\widehat{\chi}(\Phi) x(1-x),
$$

with $x=\Phi_{A} / \Phi$ the composition of polymer $A$. In the above equality, the quantity

$$
\widehat{\chi}(\Phi) \sim \chi \Phi^{\widetilde{\Delta}_{2}}
$$

accounts for the Flory effective interaction parameter between unlike blobs, where $\chi$ is the standard one, and $\widetilde{\Delta}_{2}$ some crossover exponent, of three-dimensional value $\widetilde{\Delta}_{2} \simeq 0.30$ [44]. This exponent characterizes the correction to the osmotic pressure [44]. Expression (16a) can be understood, in a certain sense, as a renormalization of interactions due to the chemical mismatch between $A$ and $B$-polymers.

Return to expression (16) and notice that it shows an obvious analogy with that defining the usual Flory-Huggins free energy of a mixture of two polymers $A$ and $B$ in the molten state $[37,38]$. The difference comes form the fact that $A$ and $B$-chains have blobs of size $\xi(\Phi)$ as new subunits, and the segregation parameter is no longer $\chi$ but the effective one $\widehat{\chi}(\Phi)$, relation $(16 a)$. Of course, these two parameters coincide in the limit $\Phi \rightarrow 1$. The model of free energy (16) constitutes the blob model [44], which is a direct consequence of the renormalization theory.

We recall now the coordinates of the demixing critical point $K$. These can be obtained equating to zero the first and second derivatives of free energy (16), with respect to composition $x$. One gets [44]

$$
\widehat{\chi}\left(\Phi_{K}, T\right)=\frac{2}{Z(\Phi)}, \quad x_{K}=\frac{1}{2},
$$

with $\Phi_{K}$ the critical monomer fraction, which is larger than the overlap one $\Phi^{*}$ defined above. The critical point $K$ is located at the top of the coexistence curve. Below $\Phi_{K}\left(\Phi<\Phi_{K}\right)$, the ternary mixture is homogeneous, while above $\Phi_{K}\left(\Phi>\Phi_{K}\right)$, this mixture phase separates in two phases alternatively rich in $A$ and $B$-polymers. Finally, we recall that the critical temperature, $T_{K}$, at fixed concentration, is given by [44]

$$
\chi\left(T_{K}\right) \sim N^{-1} \Phi^{-1 / b},
$$

with the exponent $b \simeq 0.62(d=3)$. The above relation tells us that the critical temperature $T_{K}$ should be proportional to the polymerization degree $N$ (or molecular-weight).

Now, to describe the critical phase behavior of the ternary mixture, we introduce an order parameter that is defined by

$$
x=\frac{1+\widehat{x}}{2},
$$

where $x$ is the composition of $A$-polymer in the solution. The above definition indicates that the order parameter $\widehat{x}$ is proportional to the shift $x-x_{K}$, where $x_{K}=1 / 2$ is the critical composition. The order parameter $\widehat{x}$ depends on the $d$-dimensional position-vector $\mathbf{r}=(\rho, z) \in \mathbf{R}^{d}$, with the transverse vector $\rho \in \mathbf{R}^{d-1}$ and $z \in[0, L]$ the perpendicular distance from plate 1 taken as origin. Therefore, the two plates 1 and 2 are located at $z=0$ and $z=L$, respectively. The homogeneity property of plates implies that $\widehat{x}$ depends only on the perpendicular distance $z$. We denote by $\widehat{x}_{1}$ and $\widehat{x}_{2}$ the respective values of the order parameter on plates 1 and 2 . The symmetric plates correspond to $\widehat{x}_{1}=\widehat{x}_{2}$, and asymmetric ones to $\widehat{x}_{1}=-\widehat{x}_{2}$. Since swollen $A$ and $B$-chains can be viewed as sequences of new subunits or blobs, but interact chemically through the Flory effective interaction parameter $\widehat{\chi}(\Phi)$, defined in Eq. (16), the 
total free energy (per unit area) is given by a formula similar to that defining a binary polymer mixture, relation (1). Then, we write

$$
\frac{F_{0}[\widehat{x}]}{\mathcal{A} k_{B} T}=\sum_{i=1,2}\left(-h_{i}^{0} \widehat{x}_{i}+\frac{c_{i}^{0}}{2} \widehat{x}_{i}^{2}\right)+\xi^{-d}(\Phi) \int_{0}^{L} d z\left[\frac{t_{0}}{2} \widehat{x}^{2}+\frac{u_{0}}{4} \widehat{x}^{4}+\kappa(\Phi)\left(\frac{d \widehat{x}}{d z}\right)^{2}\right]
$$

with $\mathcal{A}$ the common area of plates. Here, $t_{0}=$ $(2 / Z(\Phi)-\widehat{\chi}) / 2$ is the reduced temperature, $u_{0}=1 / 3 Z(\Phi)$ the coupling constant, $\kappa(\Phi)=\xi^{2}(\Phi) / 9$, and $\left(c_{i}^{0}, h_{i}^{0}\right)$ 's are the surface microscopic parameters relative to plates 1 and 2 . Notice that the integrand in the bulk part of the above free energy can be obtained expanding the free energy (16) to fourth order around the critical composition $x_{K}=1 / 2$. The gradient term is introduced to take into account the interfacial energy between $A$ and $B$-rich phases.

We emphasize that the above free energy is similar to that corresponding to a confined binary polymer mixture (Eq. (1)), with the simple substitutions : $a \rightarrow \xi(\Phi), N \rightarrow Z(\Phi)$. This means that chains in semi-dilute solution can be regarded as sequences of $Z(\Phi)$ blobs of the same size $\xi(\Phi)$. Taking advantage of those results derived in Sec. II, and using the above substitutions, one finds that the induced forces (per unit area) is given by

$$
\frac{\Pi_{a}^{0}}{k_{B} T_{K}}=\frac{\Delta_{\uparrow \uparrow}^{0}}{L^{4}}
$$

for symmetric (or attractive) plates, and

$$
\frac{\Pi_{r}^{0}}{k_{B} T_{K}}=\frac{\Delta_{\uparrow \downarrow}^{0}}{L^{4}}
$$

for asymmetric (or repulsive) ones, with the following universal amplitudes

$$
\begin{gathered}
\Delta_{\uparrow \uparrow}^{0}=-N a \frac{[\Gamma(1 / 4)]^{8}}{432 \pi^{2}} \Phi^{(1-v) /(3 v-1)}, \\
\Delta_{\uparrow \downarrow}^{0}=N a \frac{[\Gamma(1 / 4)]^{8}}{108 \pi^{2}} \Phi^{(1-v) /(3 v-1)} .
\end{gathered}
$$

Let us discuss these derived results.

First, we note that the above expressions obtained using the blob model, show that the presence of a good solvent simply induces a renormalization of the force amplitudes, through the multiplicative power factor $\Phi^{(1-v) /(3 v-1)} \sim \Phi^{1 / 2}$ $(d=3, v=3 / 5)$ depending on the monomer fraction $\Phi$.

Second, for both symmetric and asymmetric plates, the attractive and repulsive forces decay according to the same negative fourth power law, but with different amplitudes.

Third, as for confined polymer blends, the repulsive force is four times more important than the attractive one.

Finally, in the limit $\Phi \rightarrow 1$, the results corresponding to the molten state are then recovered.
The blob model is a mean-field theory, and it has been shown [44] that this is valid only for extremely highmolecular-weight or very high monomer concentration. To go beyond the mean-field theory, and in order to obtain correct results close to the critical point where fluctuations of composition are strong enough, use will be made of RG applied to the field theory described below.

\section{B. RG results}

The starting point is to rewrite the free energy (20) rescaling the composition fluctuation in bulk and at surfaces and parameters of the problem, according to

$$
\begin{gathered}
\varphi=\sqrt{\kappa(\Phi)}[\xi(\Phi)]^{-d / 2} \widehat{x}, \quad \varphi_{i}=\sqrt{\kappa(\Phi)}[\xi(\Phi)]_{(23 \mathrm{a})}^{-d / 2} \widehat{x}_{i}, \\
t=\frac{1}{\kappa(\Phi)} t_{0}, \quad g=\frac{6}{[\kappa(\Phi)]^{2}}[\xi(\Phi)]^{d} u_{0}, \quad(23 \mathrm{~b}) \\
c_{i}=\frac{1}{\kappa(\Phi)}[\xi(\Phi)]^{d} c_{i}^{0}, \quad h_{i}=\frac{1}{\sqrt{\kappa(\Phi)}}[\xi(\Phi)]^{d / 2} h_{i}^{0},
\end{gathered}
$$

where $\left(t_{0}, u_{0}\right)$ and $\left(c_{i}^{0}, h_{i}^{0}\right)$ 's are those parameters defined above, and $\xi(\Phi)$ the screening length. With these considerations, the total free energy reads

$$
\begin{aligned}
& \frac{F[\varphi]}{k_{B} T}=\sum_{i=1,2} \int d \rho\left(-h_{i} \varphi_{i}+\frac{c_{i}}{2} \varphi_{i}^{2}\right)+ \\
& \int d \mathbf{r}\left(\frac{1}{2}(\nabla \varphi)^{2}+\frac{t}{2} \varphi^{2}+\frac{g}{4 !}\left(\varphi^{2}\right)^{2}\right) .
\end{aligned}
$$

The field $\varphi$ depends on the spatial coordinates $\mathbf{r}=(\rho, z)$, with $\rho \in \mathbf{R}^{d-1}$ and $0 \leq z \leq L$. The quantities $\varphi_{i}$ 's are the surface fields defined on the $(d-1)$-dimensional plates 1 and 2 . The parameter $t \sim\left(T-T_{K}\right) / T_{K}$ is the reduced temperature, $g$ the coupling constant, and $\left(c_{i}, h_{i}\right)$ 's are the new surface parameters. Then, fields $\varphi$ and $\varphi_{i}$ 's, and bulk and surface parameters have the following dimensions : $[\varphi]=\left[\varphi_{i}\right]=l^{1-d / 2},[t]=l^{-2}$, $[g]=l^{d-4},\left[c_{i}\right]=l^{-1},\left[h_{i}\right]=l^{-d / 2}$, where $l$ is some length. Notice that, at the critical dimension of the system $d_{c}=4$, the coupling constant $g$ becomes marginal. 
Therefore, the theoretical model is a $\varphi^{4}$-field theory described by the above Landau-Ginzburg-Wilson Hamiltonian. Recall that the critical adsorption emerges in the limit $h_{i} \rightarrow$ $\pm \infty$.

With the help of this field model, one can compute the Casimir force. To this end, we first note that the above Hamiltonian is nothing else but that describing the critical properties of binary liquid mixtures of small molecules near the consolute point, one-component fluids near the liquid-gas critical point, or Ising-like magnetic materials near the Curie temperature. Thus, the $\varphi$-field (order parameter) may play the role of the difference between compositions for simple liquid mixtures, the difference between liquid and gas densities for one-component fluids, or the local magnetization for Isinglike magnetic materials. In this sense, the ternary mixture of our interest belongs to the universality class $(n=1, d)$, where $n$ is the number of components of the order parameter. Thus, the critical phase behavior of ternary polymer mixtures is of Ising type $[45,46]$. One can thus take advantage of some work by Krech [10], which was concerned with the computation of the Casimir force in confined liquid mixtures. To determine the force expression for confined ternary polymer mixtures, one can follow those techniques used by the author. Let us first write the Casimir force as [36]

$$
\Pi_{a, r}=\Pi_{a, r}^{0}+\delta \Pi_{a, r} .
$$

The quantity $\Pi_{a, r}^{0}$ is the mean-field force calculated above, relations (21) or (22). The remaining part, $\delta \Pi_{a, r}$, represents the force deviation due to strong fluctuations of composition. We recall that the induced force is defined through the expectation mean-value of perpendicular component of the stress tensor [10], which has been calculated using the well-known loopexpansion $[45,46]$. The mean-field contribution $\Pi_{a, r}^{0}$ represents the zeroth order of this expansion, while $\delta \Pi_{a, r}$ accounts for the contribution of higher orders.

To determinate the force deviation, the authors of Ref. [36] started form the Casimir free energy per unit area, $\delta f_{a, r}$, which can be written on the following scaling form

$$
\frac{\delta f_{a, r}}{k_{B} T}=\frac{1}{L^{d-1}} g_{a, r}\left(\frac{L}{\xi_{t}}\right) .
$$

The factor $1 / L^{d-1}$ simply expresses the natural dimension of the reduced Casimir energy $\delta f_{a, r} / k_{B} T$. Here, $\xi_{t} \sim$ $R(\Phi)[Z(\Phi)]^{-v_{t}+1 / 2}\left|1-T / T_{K}\right|^{-v_{t}}$ denotes the thermal correlation length, where $v_{t} \simeq 0.6$ is the standard Ising exponent $[45,46]$, and $R(\Phi) \sim a N^{1 / 2} \Phi^{(2 v-1) / 2(1-3 v)}$ the size of a chain in semi-dilute solution [37], with the swelling exponent $\mathrm{v} \simeq 0.588$ [47] that must not be confused with $v_{t}$. On the other hand, the scaling function $g_{a, r}(x)$ is analytic for $x<<1$ $\left(L<<\xi_{t}\right)$. Then, at the critical point $T=T_{K}\left(\xi_{t} \rightarrow \infty\right)$, $g_{a, r}(0)$ is finite and we write it as : $g_{a}(0)=\delta \Delta_{\uparrow \uparrow} /(d-1)$ or $g_{r}(0)=\delta \Delta_{\uparrow \downarrow} /(d-1)$, where $\delta \Delta_{\uparrow \uparrow}$ and $\delta \Delta_{\uparrow \downarrow}$ are the Casimir amplitudes. Then, at the critical point, the Casimir free energy decays in a universal way as

$$
\frac{\delta f_{a}}{k_{B} T_{K}}=\frac{\delta \Delta_{\uparrow \uparrow} /(d-1)}{L^{d-1}}, \quad \frac{\delta f_{r}}{k_{B} T_{K}}=\frac{\delta \Delta_{\uparrow \downarrow} /(d-1)}{L^{d-1}} .
$$

The Casimir force deviation (per unit area) $\delta \Pi_{a, r}$ is given by the first derivative of $\delta f_{a, r}$ with respect to separation $L$ : $\delta \Pi_{a, r}=-\partial \delta f_{a, r} / \partial L$. One then finds, at three dimensions,

$$
\frac{\delta \Pi_{a}}{k_{B} T_{K}}=\frac{\delta \Delta_{\uparrow \uparrow}}{L^{3}}
$$

for attractive walls, and

$$
\frac{\delta \Pi_{r}}{k_{B} T_{K}}=\frac{\delta \Delta_{\uparrow \downarrow}}{L^{3}},
$$

for repulsive ones. Notice that, in general, the force amplitudes are universal, and they depend only on space dimension $d$ and surface universality class (choice of boundary conditions). The amplitudes $\delta \Delta_{\uparrow \uparrow}$ and $\delta \Delta_{\uparrow \downarrow}$ have been calculated through a perturbative expansion with respect to the coupling constant $g$. Then, at fixed point $g^{*}$, these amplitudes become series in $\varepsilon=4-d$ ( 4 is the critical dimension of the system) that must be resummed using Borel-Leroy techniques $[45,46]$ to get their best values at dimension $d=3(\varepsilon=1)$. In three dimensions, these force amplitudes are [10]

$$
\delta \Delta_{\uparrow \uparrow} \simeq-0.652, \quad \delta \Delta_{\uparrow \downarrow} \simeq 4.48 .
$$

These values are in good agreement with MC simulation [48].

With these considerations, the total Casimir forces (per unit area) write

$$
\begin{gathered}
\frac{\Pi_{a}}{k_{B} T_{K}}=\frac{\Delta_{\uparrow \uparrow}^{0}}{L^{4}}+\frac{\delta \Delta_{\uparrow \uparrow}}{L^{3}}, \\
\frac{\Pi_{r}}{k_{B} T_{K}}=\frac{\Delta_{\uparrow \downarrow}^{0}}{L^{4}}+\frac{\delta \Delta_{\uparrow \downarrow}}{L^{3}},
\end{gathered}
$$

where the mean-field amplitudes $\Delta_{\uparrow \uparrow}^{0}$ and $\Delta_{\uparrow \downarrow}^{0}$ are those defined by relations $(22 a)$ and $(22 b)$.

Let us make some commentaries.

Firstly, Eqs. (31) and (32) indicate that, when they are reduced by the $k_{B} T_{K}$-factor, the attractive and repulsive Casimir forces $\Pi_{a, r}$ are universal, independently on the chemical structure of polymers and plates.

Secondly, we emphasize that the force expressions (31) and (32), when they are compared to those corresponding to the molten state [24], show that the solvent induces a drastic change of the force expression. Indeed, the swelling of chains modifies the dependence of the force on distance, through the appearance of the $L^{-3}$-decay. This change of the behavior is not surprising, since in the presence of a good solvent, fluctuations of composition close to the consolute point are strong enough.

Thirdly, the above formulae suggest the existence of a cross-over phenomenon occurring at some characteristic distance $L^{*}$, obtained making a comparison between the meanfield contribution $\left(\sim L^{-4}\right)$ and the fluctuation one $\left(\sim L^{-3}\right)$. This comparison gives the cross-over distance

$$
L^{*} \sim a N \phi^{(1-v) /(3 v-1)} \sim a N \phi^{1 / 2},
$$


which depends on the molecular-weight (through $N$ ) and monomer fraction $\Phi$. At the threshold $\Phi=\Phi^{*}$, the length $L^{*}$ becomes of the order of the gyration radius $R_{G} \sim a N^{v}$ of a single chain in dilute solution. At high separations $\left(L>>L^{*}\right)$, fluctuations of composition dominate, and then, the effective force behaves as $L^{-3}$. For low separations $\left(L<<L^{*}\right)$, however, a mean-field result is expected, and the effective force scales rather as $L^{-4}$. As a matter of fact, this can be understood as follows. When the distance between plates is lowered, the local monomer concentration is increased, and one assists to a strong screening of excluded volume interactions. This is why the mean-field theory works at small distances.

In Fig. 3, we superpose curves representing attractive meanfield force $\Pi_{a}^{0}$ (dashed line) and attractive fluctuations one $\delta \Pi_{a}$ (solid line), versus separation $L$. In Fig. 4, we report curves describing repulsive mean-field force $\Pi_{r}^{0}$ (dashed line) and repulsive fluctuations one $\delta \Pi_{r}$ (solid line), versus separation $L$. For the two cases, curves intersect at the cross-over distance $L^{*}$ which is different for the two boundary conditions. All these curves are drawn with parameters: $a=10$ Angstroms, $N=100, \Phi=0.5$.

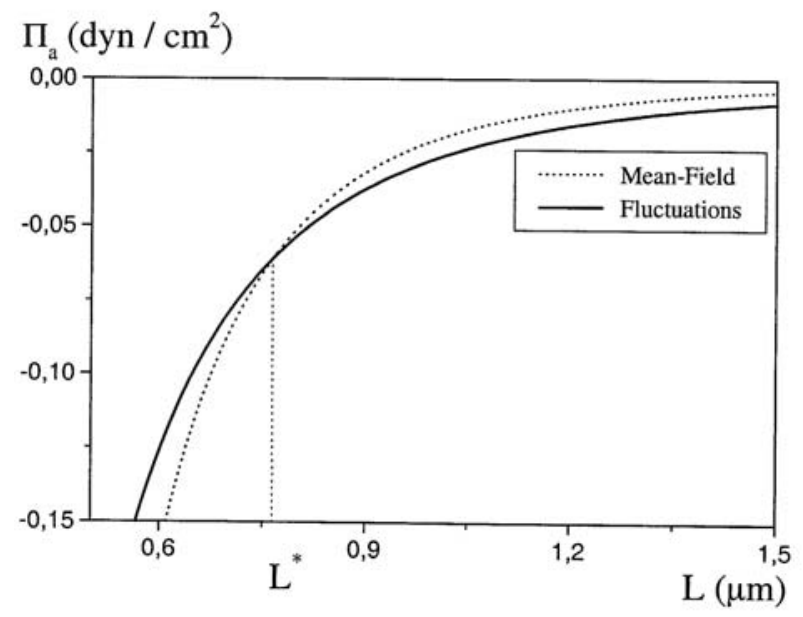

FIG. 3: Superposition of curves representing attractive mean-field force (dashed line) and attractive fluctuations force (solid line), versus separation $L$. The curves are drawn with parameters : $a=10$ Angstroms, $N=100, \Phi=0.5$.

\section{CONCLUSIONS}

In this review paper, we first considered a critical binary mixture made of two incompatible polymers $A$ and $B$, confined between two parallel plates 1 and 2 . We assumed that the latter adsorb strongly the polymers at high temperature. The fluctuations of composition generate an effective force between the two plates. We were interested in the computation of such a force. To this end, use was made of the standard $\varphi^{4}$-theory, where $\varphi$ is the composition fluctuation or order parameter. This plays the role of the magnetization of Ising-like magnetic systems. It was shown that the presence of the surfaces can be taken into account by imposing two boundary

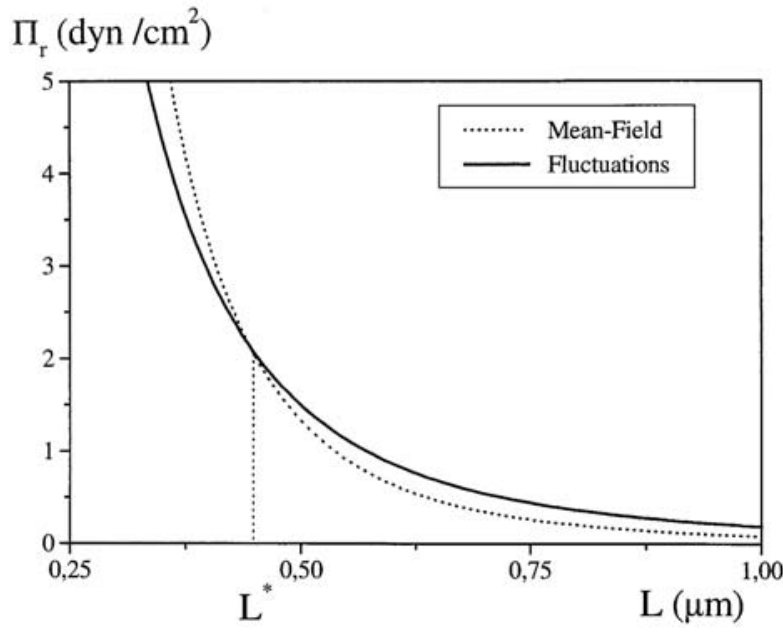

FIG. 4: Superposition of curves describing repulsive mean-field force (dashed line) and repulsive fluctuations force (solid line), versus separation $L$. These curves are drawn with the same parameters as for Fig. 3.

conditions, which are similar to the ones usually encountered in surface critical phenomena. These boundary conditions depend on two couples of parameters : $\left(c_{1}, h_{1}\right)$ for plate 1 , and $\left(c_{2}, h_{2}\right)$ for plate 2 , where $c_{1}$ and $c_{2}$ (surface enhancements) describe the interaction changes in the surface layers. The parameters $h_{1}$ and $h_{2}$ are analogous to surface magnetic fields. For the sake of simplicity, we have chosen two typical boundary conditions : symmetric and asymmetric plates, depending on whether the plates have the same or opposite preferences for the two polymers. For both symmetric and asymmetric plates, one found that the (attractive and repulsive) forces (per unit area) decrease with the separation $L$ according to the same negative power law, that is $\Pi_{r, a} \sim \pm L^{-4}$, with known exact universal amplitudes. The obtained force for critical polymer blends was compared to the electromagnetic one resulting from vacuum fluctuations of the magnetic field confined to the same geometry. Incidentally, the two kinds of forces decrease with $L$ according to the same power law.

We think that the expected force could be measured in an experiment similar to that used for measurement of the repulsive force between two plates coated by an adsorbed polymer [49].

We emphasize that since we were concerned with longpolymer chains $(N \rightarrow \infty)$, mean-field theory was sufficient to describe the phenomenon as demonstrated many years ago by de Gennes [50]. Indeed, the critical region size scales as $N^{-1}$, and then, a classical behavior should be observed in the limit $N \rightarrow \infty$.

The second purpose was a succinct recall of the determination of the Casimir force within confined ternary polymer solutions between two parallel adsorbing plates. These solutions are made of two incompatible polymers $A$ and $B$ immersed in a common good solvent. In addition to the chemical segregation between unlike chains, excluded volume interactions are present. To determine the expression of the expected force, we have restricted ourselves to two surface universality classes : 
symmetric and asymmetric plates. The induced force is attractive for symmetric plates, and repulsive for asymmetric ones. Calculations have been done, first, using the blob model. For the two boundary conditions, one found that the forces decay similarly as $L^{-4}$, and the corresponding amplitudes are similar to those relative to the molten state, up to a multiplicative power factor of the monomer fraction.

Notice that the blob model has a mean-field character, which is valid only for very strong monomer concentrations or extremely high molecular-weights. To see this, denote by $\Delta^{*} \Phi=\Phi-\Phi_{K}$ and $\Delta^{*} T=T-T_{K}$ the ranges of monomer fraction and temperature, respectively. The determination of the size of the critical region has been done using a Ginzburg criterion [44]. We sketch simply the result that : $\Delta^{*} \Phi / \Phi_{K} \sim$ $M^{-\Delta_{2} /\left(1+\Delta_{2}\right)}$ and $\Delta^{*} T / T_{K} \sim\left(\Phi_{K} / \Phi^{*}\right)^{-1 /(3 v-1)}$ with $M$ the molecular-weight, and $\Delta_{2}=\widehat{\Delta}_{2}(3 v-1) \simeq 0.22$ a cross-over exponent. Hence, in the limit of extremely long chains and very high concentrations, the above expressions suggest that the critical region is very narrow, and then, the phase behavior can be obtained using the mean-field approximation.

To go beyond the blob model, and in order to obtain a correct induced force, one applied the RG-machineries to a $\varphi^{4}$ field theory we described above. In particular, it has been shown the existence of two distance-regimes. Below some characteristic length $L^{*}$, the blob model is reliable, and the force decays as $L^{-4}$. Above $L^{*}$, however, one assists to a drastic change of the force expression due to the presence of strong fluctuations of composition. In this regime, it was found that the force decreases rather as $L^{-3}$.

We recall that the used continuous field model belongs to the same universality class as the (Ising-like) lattice model [51]. The composition fluctuation plays the role of magnetization, and the surface chemical potential exchange the surface magnetic field. Since, we are interested in phenomena occurring at criticality, these models are equivalent for the description of physics.

We point out that we focused our attention on the critical adsorption situation, only. In critical phenomena language, this corresponds to infinitely strong surface field. In this limit, the Casimir force was found to be universal. For both symmetric and asymmetric plates cases, the powers of separation $L$ are the same, but, as it should be, the corresponding universal amplitudes are numerically different. For finite values of the surface parameters (coupling constants and surface fields), however, the expected force is no longer universal, and must depend on the particular choice of the boundaries conditions.

Finally, we emphasize that, from a theoretical point of view, the Casimir force for a spherical geometry has also attracted much attention. More precisely, in recent works [52-55], the question was addressed to the computation of the induced force between colloidal particles immersed in a critical binary polymer mixture. As main result, the force decays as $r^{-3}$, where $r$ is the interparticle distance.

\section{Acknowledgments}

We would like to thank Professor Daoud for helpful discussions. We are much indebted to Professors M. Krech and S. Dietrich for useful correspondences.
[1] H.B.G. Casimir, Proc. K. Ned. Akad. Wet. 51, 793 (1948).

[2] An extensive list of recent works on the subject can found in : J. Feinberg, A. Mann, and M. Revzen, Preprint, hep-th/9908149, June 2000.

[3] S.K. Lamoureux, Phys. Rev. Lett. 78, 5 (1997).

[4] U. Mohideen and A. Roy, Phys. Rev. Lett. 81, 4549 (1998).

[5] M.E. Fisher and P.-G. de Gennes, C. R. Acad. Sci. (Paris) Série B 287, 207 (1978) ; P.-G. de Gennes, C. R. Acad. Sci. (Paris) II 292, 701 (1981).

[6] M. Krech, The Casimir Effect in Critical Systems (World Scientific, Singapore, 1994).

[7] N.M. Barber, in : Phase Transitions and Critical Phenomena, edited by C. Domb and M.S. Green, Vol. 8, (Academic Press, New York, 1983) ; E. Brézin, J. Phys. (Paris) 43, 15 (1982).

[8] V. Privman, in : Finite Size Scaling and Numerical Simulation of Statistical Systems, edited by V. Privman (World Scientific, Singapore, 1990).

[9] M. Krech and S. Dietrich, Phys. Rev. A 46, 1886 (1992).

[10] M. Krech, Phys. Rev. E 56, 1642 (1997).

[11] H.W.J. Blöte, J.L. Cardy, and M.P. Nightingale, Phys. Rev. Lett. 56, 742 (1986).

[12] I. Affleck, Phys. Rev. Lett. 56, 746 (1986).

[13] J.L. Cardy, Nucl. Phys. B 275, 200 (1986).

[14] T.W. Burkhardt and T. Xue, Phys. Rev. Lett. 66, 895 (1991) ; Nucl. Phys. B 345, 653 (1991).

[15] T.W. Burkhardt and E. Eisenriegler, Nucl. Phys. B 424 [FS],
487 (1994).

[16] K. Symanzik, Nucl. Phys. B 190 [FS], 1 (1981).

[17] M.P. Nightingale and J.O. Indeken, Phys. Rev. Lett. 54, 1824 (1985) ; J.O. Indeken, M.P. Nightingale, and W.V. Wang, Phys. Rev. B 34, 330 (1986).

[18] M. Krech and S. Dietrich, Phys. Rev. Lett. 66, 345 (1991); 67, 1055 (1981).

[19] E. Eisenriegler and M. Stapper, Phys. Rev. B 50, 10009 (1994).

[20] T.W. Burkhardt and E. Eisenriegler, Phys. Rev. Lett. 74, 3189 (1995).

[21] E. Eisenriegler and U. Ritschel, Phys. Rev. B 51, 13717 (1995).

[22] K.K. Mon, Phys. Rev. Lett. 54, 2671 (1985); M. Krech and D.P. Landau, Phys. Rev. E 53, 4414 (1996).

[23] A list or recent experimental works can be found in Refs. [6] and $[10]$.

[24] R. Cherrabi, A. Saout Elhak, M. Benhamou, and M. Daoud, Phys. Rev. E 62, 6795 (2000).

[25] D. Beysens and S. Leibler, J. Phys. Lett. (Paris) 43, L-133 (1982).

[26] C. Franck and S.E. Schnatterly, Phys. Rev. Lett. 48, 763 (1982).

[27] M. Schlossman, X.-L. Wu, and C. Franck, Phys. Rev. B 31, 1478 (1985).

[28] J.A. Dixon, M. Schlossman, X.-L. Wu, and C. Franck, Phys. Rev. B 31, 1509 (1985).

[29] An extensive list of very recent experiments using some new techniques can be found in : A. Hanke, M. Krech, F. Sch- 
lessener, and S. Dietrich, Phys. Rev. E 60, 5163 (1999).

[30] S. Blümel and G.H. Findenegg, Phys. Rev. Lett. 54, 447 (1985).

[31] E. Brézin and S. Leibler, Phys. Rev. B 27, 594 (1983) ; S. Leibler, Thesis, (Université Paris XI, 1984).

[32] J. Rudnik and D. Jasnow, Phys. Rev. Lett. 48, 1595 (1982).

[33] S. Leibler and L. Peliti, J. Phys. C 15, L-403 (1982).

[34] L. Peliti and S. Leibler, J. Phys. C 16, L-2635 (1983)

[35] Critical adsorption on curved objects, such as single spherical and rod-like colloidal particles, has been investigated in : A. Hanke and S. Dietrich, Phys. Rev. E 59, 5081 (1999) ; A. Hanke, Phys. Rev. Lett. 48, 2180 (2000).

[36] H. Ridouane, E.-K. Hachem, and M. Benhamou, Condensed Matter Physics 7, 63 (2004).

[37] P.-G. de Gennes, Scaling Concepts in Polymer Physics (Cornell University Press, Ithaca, New York, 1979).

[38] P.J. Flory, Principles of Polymer Chemistry (Cornell University Press, Ithaca, 1953).

[39] K. Binder, in : Phase Transitions and Critical Phenomena, edited by C. Domb and J.L. Lebowitz, Vol. 8, (Academic Press, London, 1983).

[40] H.W. Diehl, in : Phase Transitions and Critical Phenomena, edited by C. Domb and J.L. Lebowitz, Vol. 10, (Academic Press, London, 1986).

[41] R. Cherrabi, A. Saout Elhak, M. Benhamou, and M. Daoud, J. Chem. Phys. 111, 8174 (1999).

[42] I.S. Gradshteyn and I.M. Ryzik, Table of Integrals, Series and
Products (Academic Press, New York, 1980).

[43] V. Privman, in: Finite Size Scaling and Numerical Simulation of Statistical Systems, edited by V. Privman (World Scientific, Singapore, 1990).

[44] D. Broseta, L. Leibler, and J.-F. Joanny, Macromolecules 20, 1935 (1987).

[45] J. Zinn-Justin, Quantum Field Theory and Critical Phenomena (Clarendon Press, Oxford, 1989).

[46] C. Itzykson and J.M. Drouffe, Statistical Field Theory : 1 and 2 (Cambridge University Press, 1989).

[47] J. des Cloizeaux and G. Jannink, Polymers in Solution (Oxford University Press, Oxford, 1990).

[48] M. Krech and D.P. Landau, Phys. Rev. E 53, 4414 (1996).

[49] K. Shinozaki, Y. Saito, and T. Nose, Polymer 23, 1937 (1982).

[50] P.-G. de Gennes, J. Phys. Lett. (Paris) 38, L-441 (1977) ; J.-F. Joanny, J. Phys. A 11, L-177 (1978) ; K. Binder, J. Chem. Phys. 79, 6387 (1983).

[51] K. de Bell and T. Lookman, Rev. Mod. Phys. 65, 87 (1993).

[52] H. Ridouane, E.-K. Hachem, and M. Benhamou, J. Chem. Phys. 118, 10780 (2003).

[53] M. Benhamou, H. Ridouane, A. Derouiche, and M. Rahmoune, J. Chem. Phys. 122, 244913 (2005).

[54] E.-K. Hachem, Thesis, Hassan II-Mohammedia University, 2003.

[55] H. Ridouane, Thesis, Hassan II-Mohammedia University, 2004. 